\title{
Avaliação das Tensões Residuais em Juntas Soldadas de Inconel 625 Obtidas Através da Soldagem por Friç̧ão e Mistura Mecânica
}

\author{
Guilherme Vieira Braga Lemos ${ }^{1,2}$, Rafael Menezes Nunes², Philipp Doll2,3, Luciano Bergmann ${ }^{1}$, Telmo Roberto Strohaecker², \\ Jorge Fernandez dos Santos ${ }^{1}$ \\ 1 Helmholtz-Zentrum Geesthacht GmbH, Institute of Materials Research, Materials Mechanics, Solid State Joining Processes - \\ WMP, Geesthacht, Germany. \\ 2 Universidade Federal do Rio Grande do Sul - UFRGS, Programa de Pós-graduação em Engenharia de Minas, Metalúrgica e de \\ Materiais - PPGE3M, Porto Alegre, RS, Brasil. \\ 3 Karlsruhe Institute of Technology - KYT, Karlsruhe, Germany.
}

Recebido: 08 Abr., 2016

Aceito: 27 Abr., 2017

E-mail: guilherme.lemos@ufrgs.br (GVBL)
Resumo: A utilização de materiais nobres é requisito básico em aplicações onde existe um ambiente agressivo como na indústria do petróleo e nuclear. Neste panorama, a liga Inconel 625 é frequentemente utilizada como material de cladeamento no revestimento interno de dutos rígidos. Assim, as superligas de níquel exercem papel fundamental nos campos de exploração de águas profundas e, por isso, o conhecimento de métodos modernos de soldagem aplicados a estas ligas e suas consequências nos estados de tensões residuais é importante. Portanto, o presente trabalho faz uma avaliação das tensões residuais após a Soldagem por Fricção e Mistura Mecânica (SFMM) em chapas soldadas de Inconel 625. A união das chapas foi realizada com rotação da ferramenta 200 e 1200 rpm e velocidade de soldagem constante $(1 \mathrm{~mm} / \mathrm{s})$. As medições de tensões residuais na superfície das juntas soldadas foram investigadas através da técnica de difração de raios-X. Além disso, foram avaliadas as macroestruturas e o aporte térmico de acordo com os parâmetros de soldagem empregados. Embora não existam muitos trabalhos relacionados ao processo SFMM para ligas de Inconel, provavelmente devido à sua dificuldade de soldagem, tem sido percebido um aumento na aplicação da soldagem no estado sólido como excelente alternativa para as superligas à base de níquel. Os resultados mostraram que diferentes parâmetros de processo produziram juntas soldadas distintas e, consequentemente, variações na distribuição de tensões residuais. Por fim, um aumento na velocidade de rotação da ferramenta ocasionou um aumento nas tensões residuais na zona de mistura.

Palavras-chave: Tensões residuais; Difração de raios-X; Inconel 625; Soldagem por Fricção e Mistura Mecânica.

\section{Residual Stress Evaluation in Friction Stir Welds of Inconel 625}

\begin{abstract}
The use of noble materials is a basic requirement in applications where an aggressive environment such as offshore industry and nuclear energy. In this scenario, the Inconel 625 is often used as clad material in rigid pipes. Thus, nickel superalloys play a fundamental role in the deepwater exploration fields and, therefore, understanding of modern welding methods applied to these alloys and their consequences in the residual stress states is very important. Hence, this work evaluates the residual stress distributions after Friction Stir Welding (FSW) applied on Inconel 625 sheets. FSW was performed with tool rotational speed in the range of 200 to $1200 \mathrm{rpm}$ and welding speed constant. Measurements of superficial residual stresses were investigated by X-ray Diffraction. In addition, macrostructures and heat input were evaluated. Although there are only few reports related to FSW applied on Inconel alloys, probably due to this welding process difficulty, it has been regarded that there is cumulative application of a solid state welding as an excellent alternative for nickel superalloys. The results showed that different welding parameters produced distinctly welded joints and consequently changes in the residual stress distributions. Finally, an increase in the tool rotation speed increased the residual stress in the stir zone.
\end{abstract}

Key-words: Residual stresses; X-ray diffraction; Inconel 625; Friction Stir Welding.

\section{Introdução}

Os principais desafios da indústria do petróleo estão relacionados à exploração de poços profundos e ambientes agressivos, particularmente em áreas de águas profundas, como as da costa do Brasil e na região do Ártico [1]. Poços profundos geralmente apresentam
Este é um artigo publicado em acesso aberto (Open Access)
sob a licenca Creative Commons Attribution Non-Commercia que permite uso, distribuicão e reprodução em qualquer meio, sem restriçōes desde que sem fins comerciais eque 0 trabalho original seja corretamente citado. 
um ambiente extremamente agressivo aos componentes mecânicos e, desta maneira, exigem materiais de alto desempenho. A seleção de materiais deve garantir a resistência à corrosão, resistência máxima e confiabilidade necessária para o ambiente agressivo [2]. Assim, para atender a todos esses requisitos, superligas que contêm quantidades elevadas de $\mathrm{Ni} \mathrm{e} \mathrm{Cr}$ [3] podem ser uma ótima alternativa. Para a tecnologia submarina, tubos cladeados têm sido frequentemente usados para transporte de petróleo até a plataforma de produção. Para proteger estas tubulações contra o ambiente corrosivo composto por sal e gases como $\mathrm{H}_{2} \mathrm{~S}$ e o $\mathrm{CO}_{2}$, a utilização de uma camada resistente à corrosão é uma solução potencial. Este é o caso da aplicação de um duto rígido de aço revestido internamente com uma camada resistente à corrosão, constituída de uma superliga à base de níquel. Neste caso, a liga Inconel $625^{\circledR}$ é uma solução comum na indústria do petróleo, tratando-se de um material que possui estrutura cristalina cúbica de faces centradas (CFC) [4] com endurecimento por solução sólida. Superligas à base de níquel são usadas em diversas áreas da engenharia, tais como usinas de energia nuclear, aeronaves e outros ambientes desafiadores devido às suas excelentes propriedades de resistência e corrosão [5].

Na indústria de óleo e gás, a utilização de soldagem como etapa nos processos de fabricação de tubulações de grande comprimento é essencial. Consequentemente, um processo de união no estado sólido e com baixo aporte térmico é uma forma promissora para soldagem de superligas à base de Niquel objetivando juntas soldadas livres de defeitos. Portanto, o processo de Soldagem por Fricção e Mistura Mecânica (SFMM), também conhecido como Friction Stir Welding (FSW) [6], tem um enorme potencial para superar os desafios ainda existentes em relação à soldagem tradicional das superligas a base de níquel. No entanto, a aplicação de SFMM para materiais de elevado ponto de fusão tem sido pouco estudada até agora.

As falhas em estruturas metálicas são muitas vezes relacionadas com uma combinação de dois efeitos: tensões residuais e carregamentos aplicados. Os estados de tensões residuais podem ser prejudiciais ou benéficos, dependendo de sua natureza. As tensões residuais tem carater ordinário, ou seja, elas se combinam e somam-se com as tensões aplicadas no componente mecânico. Assim, as tensões residuais trativas encontradas em juntas soldadas podem levar a uma maior tensão efetiva e, como consequência, afetar negativamente as propriedades como vida em fadiga. Por outro lado, as tensões residuais compressivas diminuem a tensão resultante e podem ser benéficas no que diz respeito à fadiga [7]. Qualquer processo de soldagem (seja ele no estado sólido ou com fusão) vai gerar estados de tensões residuais devido aos gradientes de temperatura e/ou deformações plásticas não uniformes e mudanças microestruturais decorrentes do processo de soldagem. Sendo assim, até mesmo a soldagem no estado sólido, sendo um processo de união relativamente rápido e abaixo do ponto de fusão do material, vai ter como consequência a geração de tensões residuais [8]. As tensões residuais em juntas soldadas produzidas através da soldagem por fricção e mistura mecânica foram avaliadas diversas vezes [9-15]. No entanto, a grande maioria dos estudos está relacionada a soldas de ligas de Alumínio. Em diversos casos, a distribuição de tensões residuais longitudinais em juntas soldadas produzidas através do processo SFMM apresentam curvas com um comportamento em formato de " $\mathrm{M}$ " com valores de tensões residuais maiores na direção longitudinal do que na direção transversal [10,12,15-17]. Em direção ao metal de base, os estados de tensões residuais acabam diminuindo e são compensadas por tensões de compressão. Por fim, alguns estudos [16,18], apresentaram a possibilidade de se estimar propriedades de fadiga pelos estados de tensões residuais com a utilização da abordagem Goodman [16,18].

O estado da arte apresenta poucos estudos relacionados ao processo SFMM aplicado às superligas de níquel, especialmente a liga Inconel 625. Assim, a análise de tensões residuais em chapas soldadas de Inconel 625 obtidas por Friction Stir Welding ainda não foi profundamente estudada. Por esta razão, este tópico foi investigado para diferentes condições de soldagem. Desta forma, o conhecimento das tensões residuais superficiais em chapas soldadas de Inconel 625 é de grande importância, visto que é a superfície é quem vai estar diretamente exposta ao ambiente e a sua integridade pode ser relacionada à corrosão, desgaste, tensões residuais e vida em fadiga.

\section{Materiais e Métodos}

No presente estudo, duas chapas de Inconel $625 \mathrm{com} \mathrm{3,2} \mathrm{mm} \mathrm{de} \mathrm{espessura} \mathrm{foram} \mathrm{unidas} \mathrm{na} \mathrm{posição} \mathrm{de} \mathrm{topo}$ através da Soldagem por Fricção e Mistura Mecânica. Os dados da composição química, a tensão de escoamento e o limite de resistência à tração foram obtidos através do certificado de qualidade das chapas, e estes dados podem ser visualizados na Tabela 1. 
Avaliação das Tensões Residuais em Juntas Soldadas de Inconel 625 Obtidas Através da Soldagem por Fricção e Mistura Mecânica

Tabela 1. Composição química da liga 625 utilizada neste trabalho.

\begin{tabular}{cccccccccccc}
\hline Liga & Ni & Cr & Fe & Mo & Nb & Co & Mn & Al & Ti & Si & C \\
Inconel 625 & 60,7 & 21,7 & 4,7 & 8,6 & 3,38 & 0,03 & 0,09 & 0,13 & 0,18 & 0,18 & 0,015 \\
Norma & $>58$ & $20-23$ & $<5$ & $8-10$ & $3,15-4,15$ & $<1$ & $<0,5$ & $<0,4$ & $<0,4$ & $<0,5$ & $<0,1$
\end{tabular}

Tensão de escoamento: $512 \mathrm{MPa}^{*}$, Limite de resistência: $912 \mathrm{MPa}^{*}$

*Dados para o material de base.

Em um primeiro momento, as superfícies das chapas foram lixadas para a remoção de óxidos e impurezas. A soldagem por fricção e mistura mecânica foi realizada no instituto Helmholtz-Zentrum Geesthacht com uma máquina rígida em forma de pórtico equipada com servomotores e sistemas de controle automatizados. Uma ferramenta de Nitreto de Boro Cúbico Policristalino (PCBN), modelo Q70 [19], que consiste de cerca de 70\% de CBN, com um ombro de $25 \mathrm{~mm}$ de diâmetro e pino de $3 \mathrm{~mm}$ de comprimento foi utilizada neste estudo. $O$ tamanho inicial das chapas soldadas foi de 300 x 500 mm, mas devido a limitações de tamanho das amostras no equipamento de raios- $X$, as chapas foram previamente cortadas para as medições de tensões residuais. As chapas foram fixadas na mesa de soldagem com um sistema de clamping. O sentido de rotação da ferramenta foi anti-horário e, assim, o lado esquerdo do cordão de solda foi chamado de lado de retrocesso (RS) e o lado direito chamado de lado de avanço (AS) como pode ser observado na Figura 1. Para a obtenção dos cordões de solda, utilizou-se um ângulo de ataque da ferramenta de $1,5^{\circ}$ em relação à vertical. Por fim, uma atmosfera de argônio foi empregada em torno da ferramenta objetivando uma mínima oxidação superficial.

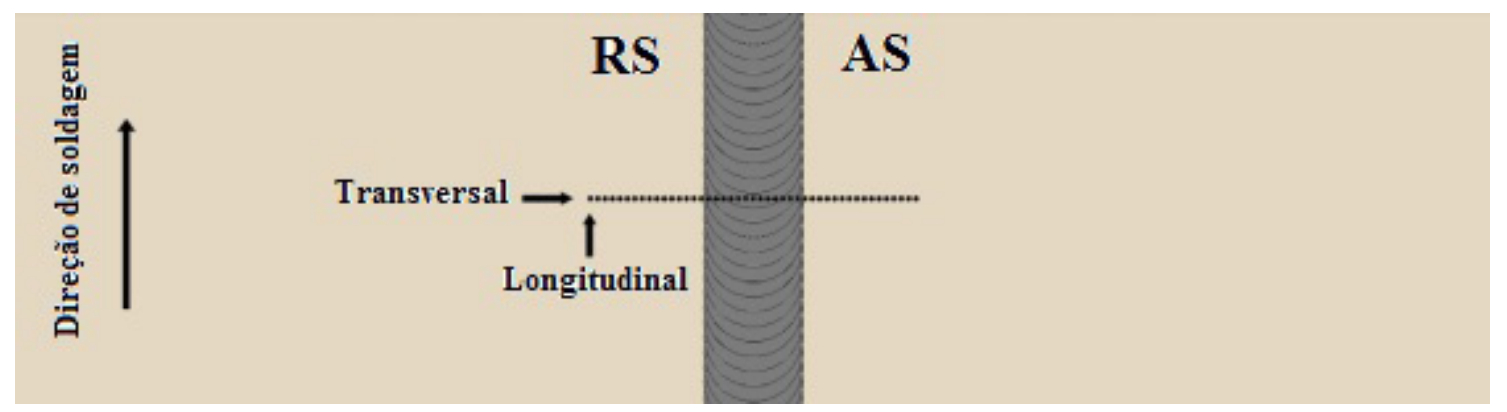

Figura 1. Esboço esquemático das posições de análise de tensões residuais. RS se refere ao lado de retrocesso e AS ao lado de avanço.

Para análise da microestrutura das juntas soldadas, as amostras foram preparadas de acordo com as práticas básicas de metalografia e, por último, atacadas com o reagente Adler [20].

\subsection{Difração de raios-X para análise de tensões residuais}

A determinação de tensões residuais a partir dos deslocamentos dos picos de difração é baseada no princípio de que quando um material é irradiado por raios-X monocromáticos para um cristal perfeitamente alinhado, os átomos são empacotados regularmente e a distância entre os planos cristalográficos é definida pelas características físicas do material. A tensão medida no retículo cristalino e as tensões residuais associadas são determinadas a partir de constantes elásticas assumindo uma distorção linear do retículo cristalino. A medida das tensões residuais pelo método de raios- $X$ é feita a partir da contribuição de vários grãos do cristal e esta medida depende fundamentalmente da geometria de feixe que irá incidir na amostra bem como do tamanho de grão da amostra.

A difração de raios-X pode ser usada para medir com precisão os espaçamentos do retículo cristalino utilizando a Lei de Bragg (Equação 1), onde n é um número inteiro, $\lambda$ é o comprimento de onda da radiação usada, $\theta$ é a metade do ângulo de espalhamento, e "d" é o espaçamento interplanar médio para uma dada reflexão de um material cristalino [21].

$$
n \lambda=2 d \operatorname{sen} \theta
$$


A determinação das tensões residuais por difração de raios-X um método diferencial onde nenhuma amostra livre de tensões residuais é necessária para determinar o estado biaxial de tensões residuais na superfície da amostra. Maiores detalhes sobre a técnica de inclinação lateral para medição de tensões residuais por difração de raios-X podem ser obtidas em [21].

Por fim, a caracterização das tensões residuais em juntas soldadas de Inconel 625 unidas através do processo Friction Stir Welding foi realizada em um difratômetro de raios-X, modelo GE-Seifert-Charon-M research edition, com geometria Bragg-Brentano e tubo de raios- $X$ de radiação $\mathrm{Cr}$-K $\alpha$ que causa uma penetração de aproximadamente $4 \mu \mathrm{m}$ em uma liga de Níquel (considerando-se $63 \%$ de penetração da radiação e um ângulo de incidência $\psi=0$ [21]), além de possuir uma repetibilidade de medição de aproximadamente 8,2 $\mathrm{MPa}$. A calibração do difratômetro foi realizada em uma amostra de pó de ferro livre de tensões. $O$ ajuste consiste em medir tensões residuais neste pó e após a avaliação de tensões faz-se ajustes nos ângulos de incidência do colimador (ângulos theta e ômega) até que os valores de tensões residuais na amostra livre de tensões sejam próximos de zero. Devido a limitações do equipamento quanto ao tamanho das amostras, as chapas foram cortadas por eletroerosão nas seguintes dimensões: $150 \times 80 \times 3,2 \mathrm{~mm}$ e, como as tensões residuais foram medidas na posição central da chapa, garantiu-se que o alívio de tensões induzido pelo corte não foi significativo na região analisada. A avaliação das tensões residuais ocorreu logo após a união das chapas, ou seja, as amostras não foram submetidas ao método de remoção de camadas. Foi utilizado um feixe difratado a partir de um colimador com abertura primária de $2 \mathrm{~mm}$ e um detector linear de 20 ㅇ GE-Meteor-1D. Os picos de difração na direção $\{220\}$ em $2 \theta=128,78$ o foram avaliados e foi considerado o método de inclinação lateral $\sin 2 \psi$ para o estudo das tensões residuais. Neste contexto, a faixa de medição em $2 \theta$ foi de $120^{\circ}$ a $139^{\circ}$ em passos de $0,1^{\circ} \mathrm{com}$ tempos de medição de 20 s por passo. A inclinação de uma regressão linear, obtida através dos pontos de dados medidos, resulta no valor da tensão residual. $\mathrm{O}$ desvio padrão das medições de tensão residual decorre de desvios de raios- $X$ posições de linha de difração em 21 posições angulares diferentes para a linha de regressão calculada com ângulos de inclinação entre -60 e $+60^{\circ}$. Finalmente, para o cálculo das tensões residuais, utilizou-se valores das constantes elásticas $1 / 2$ s2 -s1 de 6,19*10-6 $\mathrm{MPa}^{-1}$ e -1,36* 10-6 $\mathrm{MPa}^{-1}$, respectivamente [22]. O movimento automático dos eixos $\mathrm{X}, \mathrm{Y}$ e $\mathrm{Z}$ do difratômetro permitiu medições consecutivas, tal como indicado na Figura 1. Deste modo, os campos de tensões residuais foram analisados com um número total de até 82 medições por amostra. De maneira complementar, também foram caracterizados os estados de tensões residuais iniciais das chapas na condição de como recebidas do fabricante (e antes da soldagem), onde se notaram tensões residuais compressivas e homogêneas na ordem de -600 MPa.

\section{Resultados e Discussões}

\subsection{Parâmetros de soldagem}

Duas juntas soldadas de Inconel 625 foram unidas pelo processo SFMM e nomeadas como: amostra I e amostra II. A velocidade de deslocamento (velocidade de soldagem) se manteve constante $(1 \mathrm{~mm} / \mathrm{s})$, a força axial foi de $60 \mathrm{kN}$ e a velocidade de rotação da ferramenta variou de 1200 a $200 \mathrm{rpm}$ conforme dados apresentados na Tabela 2. Estes valores de velocidade de rotação foram escolhidos na seleção de parâmetros ótimos de soldagem, ou seja, estes dois parâmetros de processo foram selecionados dentro de um grupo maior de amostras. A penetração da junta soldada foi calculada com base nas metalografias e auxílio do programa Image J. Ainda, notou-se uma evolução do desgaste da ferramenta ao longo do processo de soldagem, fato que pode explicar a variação da profundidade de penetração das juntas soldadas. De fato, a falta de penetração em juntas soldadas de Inconel 625 produzidas através do processo de SFFM é comumente observada na literatura [23-29]. Para a junta soldada

Tabela 2. Visão geral dos parâmetros de soldagem empregados.

\begin{tabular}{cccccc}
\hline Amostra & $\begin{array}{c}\text { Velocidade de } \\
\text { deslocamento, } \\
\mathbf{m m} / \mathbf{s}\end{array}$ & Força axial, $\mathbf{k N}$ & $\begin{array}{c}\text { Velocidade de } \\
\text { rotação, } \boldsymbol{r p m}\end{array}$ & $\begin{array}{c}\text { Qualidade } \\
\text { superficial da } \\
\text { solda }\end{array}$ & $\begin{array}{c}\text { Profundidade de } \\
\text { penetração, } \mathbf{m m}\end{array}$ \\
I & 1 & 60 & 1200 & Média & 2,11 \\
II & 1 & 60 & 200 & Excelente & 1,81 \\
\hline
\end{tabular}


Avaliação das Tensões Residuais em Juntas Soldadas de Inconel 625 Obtidas Através da Soldagem por Fricção e Mistura Mecânica

produzida com alta velocidade de rotação da ferramenta (amostra I), a superfície apresentou uma coloração avermelhada devido ao excessivo aporte térmico. Além disso, notou-se uma heterogeneidade ao longo desta superfície da solda (Figura 2), indicadas pelas setas. Este acabamento superficial não homogêneo pode afetar significativamente as tensões residuais e a microestrutura do material [30,31] e também a resistência à fadiga de juntas soldadas. Um recente estudo complementar ao presente trabalho [24], para as mesmas chapas soldadas mostrou a presença de porosidades para a amostra $I$, fato que comprova uma pior qualidade desta junta soldada. Por fim, a junta soldada que resultou em um baixo aporte térmico, mostrada na Figura 3 (amostra II), apresentou excelente qualidade da superfície da solda, ausência de porosidades e microestrutura mais homogênea [24], sendo assim escolhida a melhor condição de soldagem.

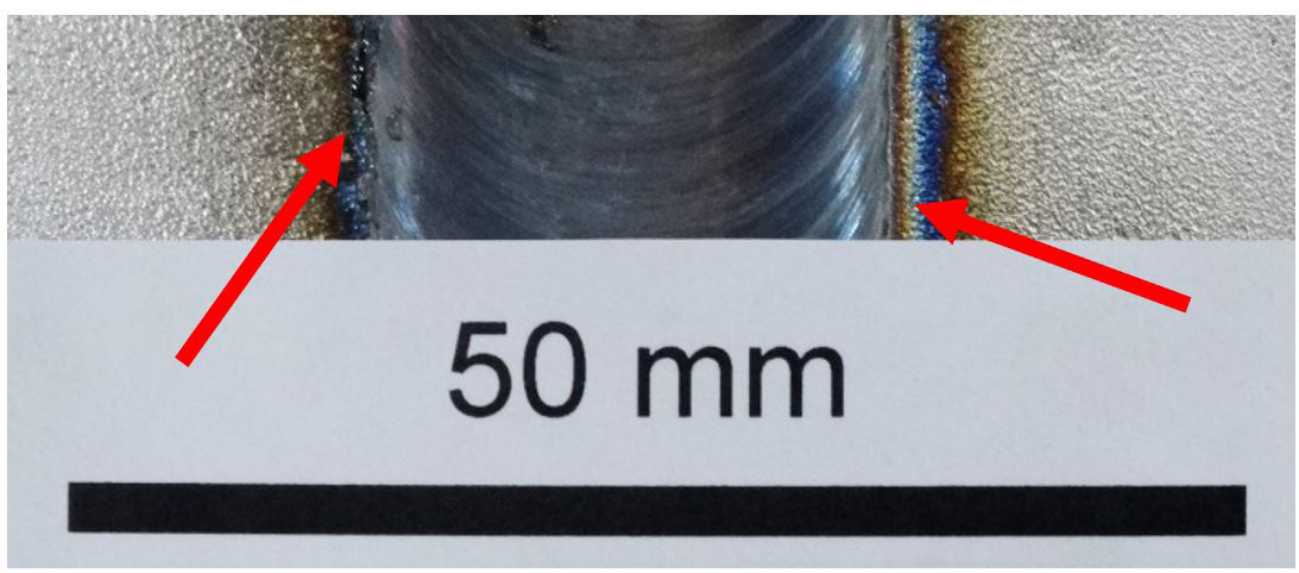

Figura 2. Aparência da superfície superior da junta soldada (amostra I). As setas indicam heterogeneidades na solda.

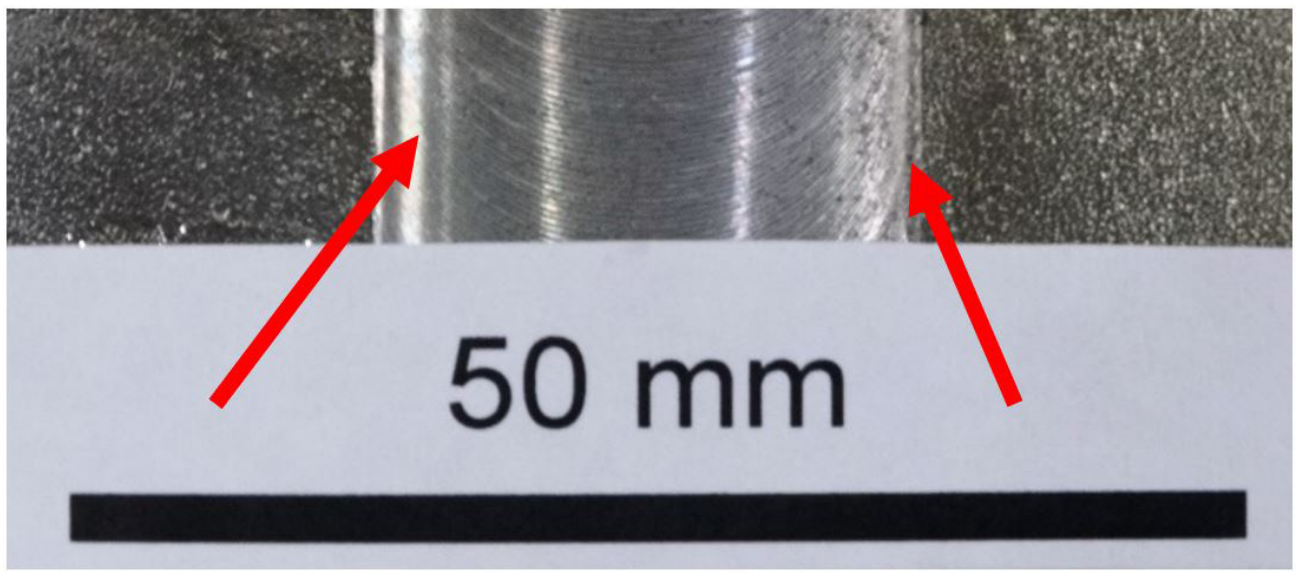

Figura 3. Aparência da superfície superior da junta soldada (amostra II). As setas indicam heterogeneidades na solda.

Nas Figura 4 e 5 são apresentadas macrografias para a seção transversal das juntas soldadas. De modo geral, observou-se que a soldagem por fricção e mistura mecânica levou a um refino de grão nas chapas soldadas quando comparadas ao material de base. A profundidade de penetração foi pelo desgaste durante a soldagem. Ainda, uma alta velocidade de rotação da ferramenta $(1200 \mathrm{rpm})$ provocou uma menor largura do cordão de solda na seção transversal e um fluxo de material heterogêneo. Além disso, notou-se que alguns grãos não foram completamente recristalizados (Figura 4). Por outro lado, uma menor velocidade de rotação ocasionou um fluxo de material mais homogêneo na seção transversal da solda e uma maior região de refino de grão próxima à superfície (Figura 5). 


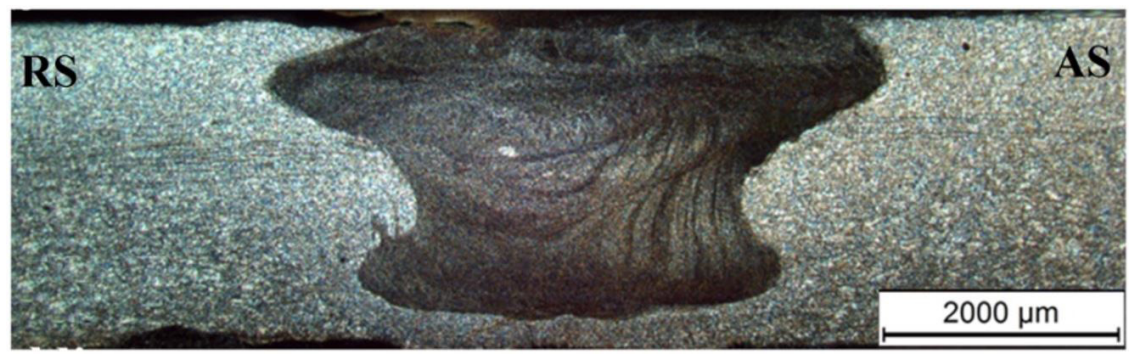

Figura 4. Macroestrutura da seção transversal da junta soldada (amostra I). RS se refere ao lado de retrocesso e AS ao lado de avanço.

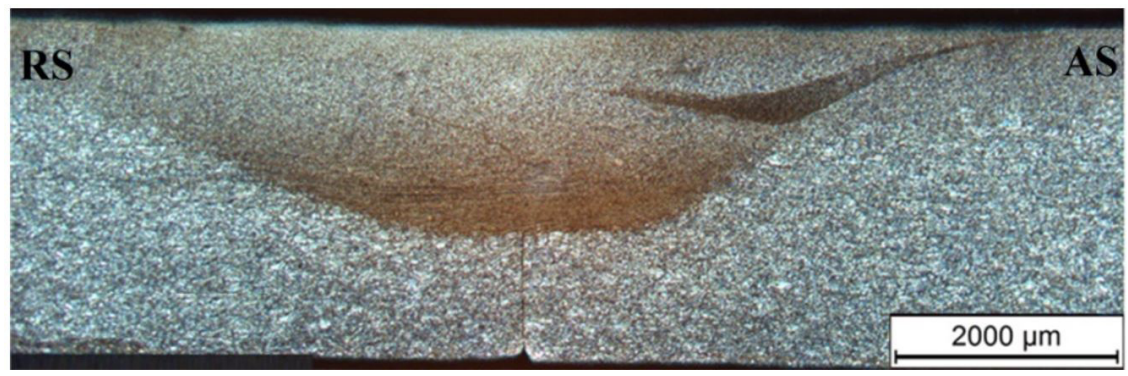

Figura 5. Macroestrutura da seção transversal da junta soldada (amostra II). RS se refere ao lado de retrocesso e AS ao lado de avanço.

\subsection{Aporte térmico}

Com base nas condições de soldagem avaliadas no presente estudo, o aporte térmico foi calculado em função da velocidade de rotação e do torque [32] usando as seguintes Equações 2 e 3:

$$
\begin{aligned}
& H I=\frac{P}{v} \\
& P=\frac{2 \pi \Omega T}{60}
\end{aligned}
$$

Onde $\mathrm{P}$ é a potência em $\mathrm{kW}, \Omega$ é a velocidade de rotação em rev/min, T é o torque do fuso da máquina FSW em $\mathrm{Nm}$, HI é o aporte térmico $(\mathrm{J} / \mathrm{mm})$ e v é a velocidade de soldagem $(\mathrm{mm} / \mathrm{seg})$. Assim, os valores de aporte térmico são apresentados na Tabela 3. Uma velocidade de rotação de ferramenta maior (1200 rpm) ocasiona um aumento do aporte térmico. De fato, a extensão da deformação é proporcional ao gradiente térmico imposto à junta soldada. Ou seja, teoricamente, quanto maior o aporte térmico, maior é a temperatura máxima atingida durante a soldagem e, consequentemente, maiores tensões trativas residuais alcançadas. Em outras palavras, processos de união que alcançam alto aporte térmico (por exemplo, arco submerso) tendem a apresentar tensões residuais mais trativas do que processos de soldagem com baixo aporte térmico (por exemplo, a soldagem a laser). De modo similar, estas comparações podem ser aplicadas dentro de um mesmo processo, ou seja, juntas soldadas produzidas

\begin{tabular}{|c|c|c|c|c|}
\hline Amostra & $\begin{array}{c}\text { Velocidade } \\
\text { soldagem, } \mathrm{mm} / \mathrm{s}\end{array}$ & $\begin{array}{l}\text { Velocidade de } \\
\text { rotação, Rpm }\end{array}$ & Torque, $\mathrm{Nm}$ & $\begin{array}{c}\text { Aporte térmico, } \\
\mathrm{kJ} / \mathrm{mm}\end{array}$ \\
\hline$I$ & 1 & 1200 & 30 & 3,77 \\
\hline II & 1 & 200 & 110 & 2,30 \\
\hline
\end{tabular}

Tabela 3. Aporte térmico como função da velocidade de rotação e torque. 
Avaliação das Tensões Residuais em Juntas Soldadas de Inconel 625 Obtidas Através da Soldagem por Fricção e Mistura Mecânica

no estado sólido (Friction Stir Welding), mas com alto aporte térmico poderão resultar em tensões residuais mais trativas quando comparadas as distribuições de tensões residuais de soldas que alcançaram baixo aporte térmico.

\subsection{Análise de tensões residuais por difração de raios-X}

As análises de tensões residuais superficiais nos sentidos longitudinal e transversal para os parâmetros de processo de soldagem estudados neste trabalho são apresentadas nas Figuras 6 e 7, respectivamente. Três regiões principais foram avaliadas, são elas: o lado RS, a linha central da solda (também chamada de zona de mistura ou coordenada $0 \mathrm{~mm}$ ) e o lado AS.

As distribuições de tensões residuais no sentido longitudinal em relação à junta soldada são mostradas na Figura 6. De modo geral, as tensões para o conjunto de amostras / e // mantêm-se em um patamar constante até aproximadamente $15 \mathrm{~mm}$ do centro da solda e, logo após esta distância, as tensões residuais iniciam uma mudança de comportamento tornando-se compressivas. De fato, as chapas de Inconel 625 já apresentavam tensões residuais compressivas e homogêneas, conforme mostrado nos estados de tensões do material de base (devido a processos de fabricação prévios a soldagem). Para a zona de mistura, chamada de linha central da solda (coordenada 0 mm),

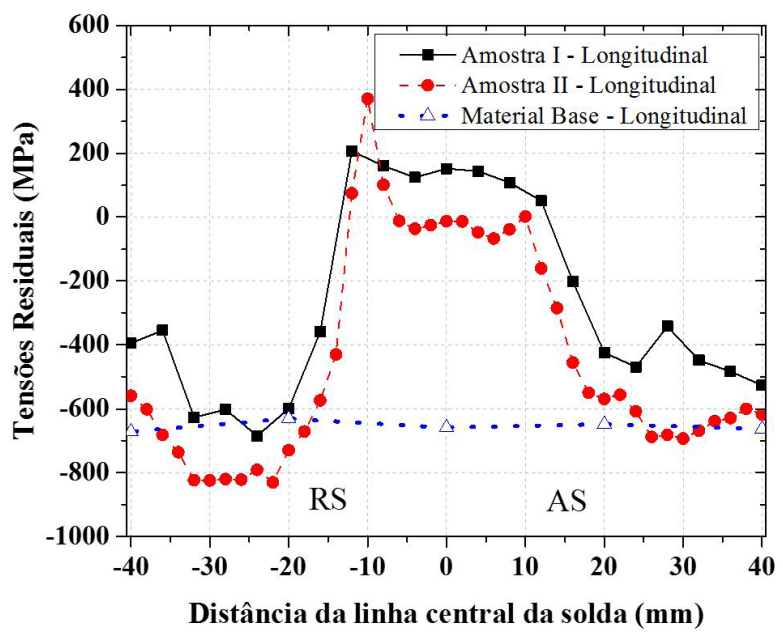

Figura 6. Distribuição de tensões residuais longitudinais em função da distância da linha central da solda. RS se refere ao lado de retrocesso e AS ao lado de avanço.

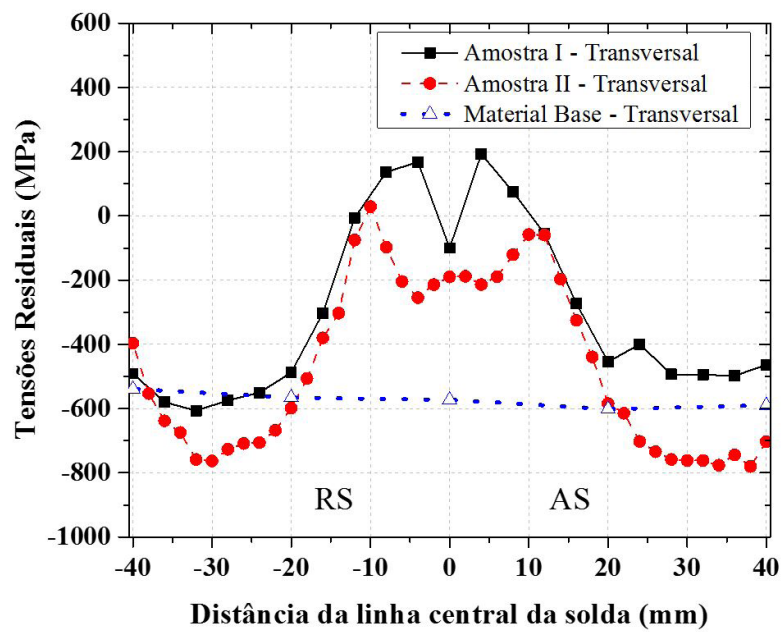

Figura 7. Distribuição de tensões residuais transversais em função da distância da linha central da solda. RS se refere ao lado de retrocesso e AS ao lado de avanço. 
a amostra I alcançou níveis de tensões trativos próximos a 200 MPa. Para uma faixa de distâncias de -12 a +14 mm, as tensões residuais alcançadas pela amostra I são maiores e trativas, em média. Em contraste, a amostra II atingiu valores de tensões residuais menores, em média, e em algumas posições específicas os valores foram nulos ou compressivos. Porém, para a região entre -10 e -14 mm de distância em relação ao centro da solda, a amostra // exibiu um pico de tensões residuais trativas com níveis próximos a $400 \mathrm{MPa}$. Conforme já relatado em outros estudos [8,15], as tensões residuais trativas máximas foram encontradas no sentido longitudinal de medições.

Os níveis das tensões residuais transversais são mostrados na Figura 7. Como pode ser observado, as tensões residuais transversais apresentaram comportamento compressivo para ambas as condições de soldagem com níveis de tensões variando-se de -150 a -200 MPa. Em média, para uma faixa de distâncias de -12 a +14 mm, os valores de tensões residuais alcançados pela amostra I são maiores que os valores obtidos pela amostra II. Ainda, a amostra II apresentou níveis mínimos de tensões residuais quando comparados à amostra $I$. As tensões residuais transversais para a amostra I apresentam um acréscimo trativo de aproximadamente $200 \mathrm{MPa}$ nas distâncias de $\pm 5 \mathrm{~mm}$ em relação ao centro da solda. Por outro lado, a amostra /l mostrou um leve incremento trativo nos níveis de tensões residuais até uma distância de $\pm 10 \mathrm{~mm}$ em relação ao centro da solda. Para os dois parâmetros de soldagem estudados, após esse incremento trativo nos níveis de tensões residuais, observa-se que existe uma tendência a estados de tensões compressivos na superfície.

Observa-se que os perfis de tensões residuais superficiais mudam em função da velocidade de rotação da ferramenta, pois foram observadas diferenças expressivas nos níveis de tensões residuais para as duas juntas soldadas. Sabe-se que a soldagem por fricção e mistura mecânica é um processo assimétrico em termos de distribuição de temperatura e deformações plásticas e, desta maneira, já eram esperados resultados de distribuição de tensões residuais distintos para os lados RS e AS, o que de fato ocorreu. Independente da direção de tensões residuais avaliada (longitudinal ou transversal), a amostra II alcançou os menores valores de tensões residuais. Além disso, estes valores foram mais compressivos (negativos), em média. Por outro lado, as tensões residuais transversais têm valor trativo máximo para a amostra I (junta soldada que atingiu o maior aporte térmico devido a maior velocidade de rotação), fato que sugere uma relação entre o maior o aporte térmico e as tensões residuais trativas transversais no centro da solda (coordenada $0 \mathrm{~mm}$ ).

Comparando-se os resultados obtidos na análise de tensões residuais longitudinais (Figura 6) com a aparência superficial das soldas, mostrada na Figura 2, notou-se que existe uma assimetria na distribuição de tensões residuais, assim como na aparência da superfície de topo das juntas soldadas ao longo da direção longitudinal. Sabe-se que os lados AS e RS exibem diferentes aportes térmicos, e esta assimetria leva a diferenças microestruturais e propriedades mecânicas locais distintas [33] e, como consequência, também existirão variações nas tensões residuais. Ainda, as Figura 4 e 5 mostram as análises macroscópicas das soldas, onde também são observadas diferenças significativas para as condições de soldagem analisadas. Estas diferenças de aporte térmico características deste processo de soldagem contribuem para mudanças na distribuição de tensões residuais principalmente no sentido longitudinal de medições.

Por fim, notou-se que existe um estado de tensões residuais prévio a soldagem devido a diferentes processos como: a laminação a frio, o recozimento e o próprio preparo das chapas com lixamento abrasivo que geraram um nível de tensões residuais no material de base. Além destes processos, o próprio "clamping system" adotado neste processo de soldagem, impõe certa restrição das deformações que pode influenciar significativamente os resultados de tensões residuais [9]. Assim, todos esses fatores influenciaram a distribuição final de tensões residuais nas chapas soldadas e os níveis de heterogeneidade destas tensões têm relação direta com todos estes processos.

\subsection{Estimativa da vida em fadiga}

A maioria das trincas de fadiga se inicia nas regiões superficiais ou subsuperficiais [30,31]. De fato, a superfície é a área que suporta a maior carga aplicada durante a utilização do componente, sendo assim susceptível a condições adversas. Além disso, ela pode também conter defeitos e tensões residuais provenientes dos processos de fabricação [18]. Neste contexto, o presente estudo considera que a amostra II apresentou melhor aparência superficial, microestrutura mais homogênea e sem porosidades [25,34] e também atingiu valores de tensões residuais mais compressivos, fatos que sugerem um melhor comportamento em fadiga. Portanto, assume-se que esta amostra tende a apresentar maior resistência à fadiga quando comparada a amostra I. Em geral, uma menor resistência à fadiga corresponde às amostras com tensões residuais longitudinais trativas elevadas [15]. 
Avaliação das Tensões Residuais em Juntas Soldadas de Inconel 625 Obtidas Através da Soldagem por Fricção e Mistura Mecânica

Para a construção de uma curva de Wöhler (curva S-N) são necessárias varias amostras e uma grande quantidade de ensaios, além do custo e tempo associados. Contudo, existem maneiras alternativas para uma simples estimativa do potencial das tensões residuais nas propriedades de fadiga como a abordagem de Goodman [15,18,35] descrita pela Equação 4. Neste contexto, uma redução na resistência à fadiga ( $\sigma a)$ é relacionada à tensão média $(\sigma \mathrm{m})$ e a resistência máxima do material ( $\sigma U T S)$. Para esta relação é coerente admitir-se que oa diminui com um aumento da $\sigma \mathrm{m}$. O limite de fadiga (sem influência de tensões residuais) é dado por $\sigma 0$. De modo similar [15,18], alguns estudos já apresentaram a relação entre as tensões residuais e vida em fadiga para o processo SFMM. Outro método disponível para se estimar a vida em fadiga é a relação parabólica de Gerber [35] conforme a Equação 5. É importante notar-se que a relação de Goodman é mais utilizada porque é mais conservadora quando comparada a abordagem de Gerber. Este fato é fácil de ser percebido através da análise da área abaixo da curva de Goodman, que é menor quando comparada a área abaixo da curva de Gerber e, consequentemente, nota-se uma menor região onde se acredita que a fadiga não ocorra.

$$
\begin{aligned}
& \sigma a=\sigma 0\left(1-\frac{\sigma m}{\sigma U T S}\right) \\
& \sigma a=\sigma 0\left(1-\left(\frac{\sigma m}{\sigma U T S}\right) 2\right)
\end{aligned}
$$

De acordo com a literatura disponível e as propriedades mecânicas apresentadas pelo certificado do fabricante do material, os valores utilizados para construção do diagrama de Haigh (que engloba tanto a reta de Goodman quanto à curva Gerber) são apresentados na Tabela 4. A resistência a fadiga $\sigma 0$ foi calculada de acordo com a relação $\sigma 0=0,25 \sigma U T S$ [36]. Assim, a Figura 8 mostra o diagrama de Haigh e a esperada redução na vida em fadiga em função de tensão média. A linha sólida azul com comportamento linear decrescente representa a relação de Goodman e a linha sólida vermelha com comportamento parabólico representa a abordagem de Gerber com ambas descrevendo a situação do material de base. Por outro lado, as linhas pontilhadas com comportamento decrescente mostram as situações da junta soldada de Inconel 625. Através da reta vertical é possível, de modo similar a estudos disponíveis na literatura, analisar a tensão média de Von Mises calculada com base nas tensões residuais trativas medidas (considerando a menor e a maior tensão residual trativa). Portanto, utilizando a

Tabela 4. Propriedades mecânicas do material de base e da amostra II.

\begin{tabular}{ccc}
\hline Condições de soldagem & Limite de resistência à fadiga (MPa) & Resistência máxima a tração (MPa) \\
Material de base & 280 & 912 \\
Amostra ll & - & 1152 (zona de mistura) [26]
\end{tabular}

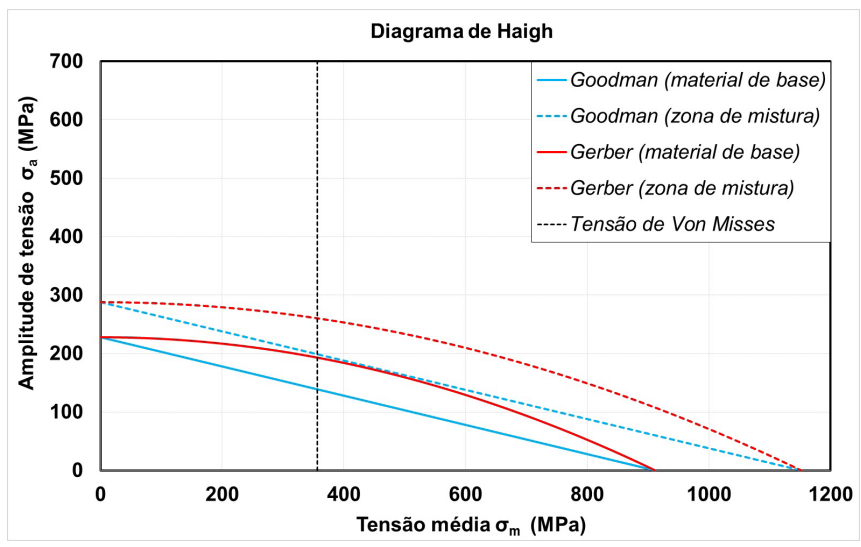

Figura 8. Diagrama de Haigh para o material de base e a solda de Inconel 625 (amostra II). 
tensão média de Von Mises como referência, percebe-se um aumento da amplitude de tensão do material de base para as chapas soldadas (para as duas abordagens). Em outras palavras, este comportamento sugere que a soldagem por friç̧ão e mistura mecânica tende a aumentar a vida em fadiga da liga Inconel 625. Deve ser notado, entretanto, que o processo Friction Stir Welding pode formar uma microestrutura complexa com grão refinado e estas simples abordagens estimativas podem não descrever de maneira completa estas características. Além das regiões de tensões residuais trativas, também existem regiões com tensões compressivas que podem retardar o crescimento de trincas de fadiga.

\section{Conclusões}

Juntas soldadas de Inconel 625 com diferentes aportes térmicos, obtidas pelo processo de soldagem e mistura mecânica, foram avaliadas no presente estudo. Os resultados das análises de tensões residuais podem ser resumidos como:

- A velocidade de rotação afetou significativamente os estados de tensões residuais. A amostra processada com maior velocidade de rotação da ferramenta apresentou um maior gradiente entre as tensões residuais na linha central da solda e os valores alcançados pelo metal de base. Na zona de mistura (coordenada $0 \mathrm{~mm}$ ), as tensões residuais podem ter um comportamento trativo ou levemente compressivo, dependendo dos parâmetros de soldagem empregados. Em geral, a junta soldada com melhor aparência da superfície de topo alcançou estados de tensões residuais mais compressivos (em média) e estes tendem a acarretar em melhores propriedades de fadiga.

- As abordagens de Goodman e de Gerber sugerem um aumento nas propriedades de fadiga devido à aplicação da Soldagem por Fricção e Mistura Mecânica na liga Inconel 625.

\section{Agradecimentos}

Os autores agradecem o Conselho Nacional de Desenvolvimento Científico e Tecnológico (CNPq) através do programa Ciência sem Fronteiras e CAPES, assim como o Instituto Helmholtz-Zentrum Geesthacht GmbH (Germany) pelo suporte para execução deste trabalho.

\section{Referências}

[1] Rolls-Royce Holdings. Annual report 2012. London: Rolls-Royce Holdings plc; 2012.

[2] McCoy S, Hereford U, Puckett B, Hibner E, Huntington W. High performance age-hardenable nickel alloys solve problems in sour oil and gas service. Balance. 2002;14:16.5.

[3] Abioye TE, McCartney DG, Clare AT. Laser cladding of Inconel 625 wire for corrosion protection. Journal of Materials Processing Technology. 2015;217:232-240. https://doi.org/10.1016/j. jmatprotec.2014.10.024.

[4] Paul CP, Ganesh P, Mishra SK, Bhargava P, Negi J, Nath AK. Investigating laser rapid manufacturing for Inconel-625 components. Optics \& Laser Technology. 2007;39(4):800-805. https://doi.org/10.1016/j.optlastec.2006.01.008.

[5] Pollock TM, Tin S. Nickel-based superalloys for advanced turbine engines: chemistry, microstructure, and properties. Journal of Propulsion and Power. 2006;22(2):361-374. http://dx.doi. org/10.2514/1.18239.

[6] Thomas WM, Nicholas ED, Needham JC, Murch MG, TempleSmith P, Dawes CJ. Friction-Stir Butt Welding, GB Patent No. 9125978.8, International patent application No. PCT/GB92/02203, 1991.

[7] Suominen L, Khurshid M, Parantainen J. Residual stresses in welded components following post-weld treatment methods. Procedia Engineering. 2013;66:181-191. http://dx.doi.org/10.1016/j. proeng.2013.12.073.
[8] Kumar N, Mishra R, Baumann J. Residual stresses in friction stir welding. Oxford: Butterworth-Heinemann; 2013. (Friction Stir Welding and Processing Book Series).

[9] Richter-Trummer V, Suzano E, Beltrão M, Roos A, Santos JF, Castro PM. Influence of the FSW clamping force on the final distortion and residual stress field. Materials Science and Engineering A. 2012;538:81-88. http://dx.doi.org/10.1016/j. msea.2012.01.016.

[10] Steuwer A, Barnes SJ, Altenkirch J, Johnson R, Withers PJ. Friction stir welding of HSLA-65 steel: part II. The influence of weld speed and tool material on the residual stress distribution and tool wear. Metallurgical and Materials Transactions. A, Physical Metallurgy and Materials Science. 2012;43(7):23562365. http://dx.doi.org/10.1007/s11661-011-0643-x.

[11] Terasaki T, Akiyama T. Mechanical behaviour of joints in FSW: Residual stress, inherent strain and heat input generated by friction stir welding. Welding in the World. 2003;47(11-12):2431. http://dx.doi.org/10.1007/BF03266405.

[12] Peel M, Steuwer A, Preuss M, Withers PJ. Microstructure, mechanical properties and residual stresses as a function of welding speed in aluminium AA5083 friction stir welds. Acta Materialia. 2003;51(16):4791-4801. http://dx.doi.org/10.1016/ S1359-6454(03)00319-7.

[13] Reynolds AP, Tang W, Gnaupel-Herold T, Prask H. Structure, properties, and residual stress of $304 \mathrm{~L}$ stainless steel friction stir welds. Scripta Materialia. 2003;48(9):1289-1294. http:// dx.doi.org/10.1016/S1359-6462(03)00024-1. 
Avaliação das Tensões Residuais em Juntas Soldadas de Inconel 625 Obtidas Através da Soldagem por Fricção e Mistura Mecânica

[14] Staron P, Kocak M, Williams S, Wescott A. Residual stress in friction stir-welded Al sheets. Physica B, Condensed Matter. 2004;350(1):E491-E493. http://dx.doi.org/10.1016/j. physb.2004.03.128

[15] Brewer LN, Bennett MS, Baker BW, Payzant EA, Sochalski-Kolbus LM. Characterization of residual stress as a function of friction stir welding parameters in Oxide Dispersion Strengthened (ODS) steel MA956. Materials Science and Engineering A. 2015;647:313321. http://dx.doi.org/10.1016/j.msea.2015.09.020.

[16] Khandkar MZ, Khan JA, Reynolds AP, Sutton MA. Predicting residual thermal stresses in friction stir welded metals. Journal of Materials Processing Technology. 2006;174(1):195-203. http:// dx.doi.org/10.1016/j.jmatprotec.2005.12.013.

[17] Buffa G, Fratini L, Pasta S. Residual stresses in friction stir welding: numerical simulation and experimental verification. In: International Centre for Diffraction Data. Proceedings of the ICRS-8 8th International Conference on Residual Stresses.; Denver, Colorado, USA. 2008. p. 444-453.

[18] Lemmen HJK, Alderliesten RC, Benedictus R. Benedictus RFatigue initiation behaviour throughout friction stir welded joints in AA2024-T3. International Journal of Fatigue. 2010;32(12):19281936. http://dx.doi.org/10.1016/j.ijfatigue.2010.06.001.

[19] Perrett J, Martin J, Peterson J, Steel R, Packer S. Friction stir welding of industrial steels. In: Mishra R, Mahoney MW, Sato Y, Hovanski Y, Verma R, editors. Friction stir welding and processing VI. Hoboken: John Wiley \& Sons, Inc, 2011. p. 65-72. http:// dx.doi.org/10.1002/9781118062302.ch9.

[20] Petzow G. Metallographisches Atzen (Materialkundlich-technische Reihe). Stuttgart: Gebruder Borntraeger Berlin; 1976.

[21] Hauk V. Structural and residual stresses analysis by nondestructive methods. Aachen: Elsevier; 1997

[22] Prevéy PS. X-ray diffraction residual stress techniques. In: Mills, K. editor. Metals handbook. 9th ed. Materials Park: American Society for Metals; 1986. v. 10, p. 380-392.

[23] Lemos GVB, Simoni L, Bergmann L, Souza D, Araujo DB, Santos JF, et al. Caracterização preliminar da microestrutura em cordões de solda da liga 625 produzidos através dos processos de SFMM e MIG. In: Associação Brasileira de Soldagem. Anais do $41^{\circ}$ Congresso Nacional de Soldagem; 2015; Salvador, Brasil. São Paulo: ABS Soldagem; 2015.

[24] Lemos GVB, Farina AB, Martinazzi D, Huppes RM, Bergmann L, Santos JF, et al. Efeito da velocidade de rotação da ferramenta na soldagem por fricção e mistura mecânica da liga inconel 625. In: Associação Brasileira de Metalurgia, Materiais e Mineração. Anais do $71^{\circ}$ Congresso Anual ABM; 2016 Set 27-29; Rio de Janeiro, Brasil. São Paulo: ABM; 2016.

[25] Song KH, Nakata K. 'Effect of precipitation on post-heattreated Inconel 625 alloy after friction stir welding'. Materials
\& Design. 2010;31(6):2942-2947. http://dx.doi.org/10.1016/j. matdes.2009.12.020

[26] Song KH, Nakata K. Mechanical properties of friction-stir-welded Inconel 625 alloy. Materials Transactions. 2009;50(10):24982501. http://dx.doi.org/10.2320/matertrans.M2009200.

[27] Song KH, Nakata K. Microstructural and mechanical properties of friction-stir-welded and post-heat-treated Inconel 718 alloy. Journal of Alloys and Compounds. 2010;505(1):144-150. http:// dx.doi.org/10.1016/j.jallcom.2010.06.016.

[28] Sato YS, Arkom P, Kokawa H, Nelson TW, Steel RJ. Effect of microstructure on properties of friction stir welded Inconel Alloy 600. Materials Science and Engineering A. 2008;477(12):250-258. http://dx.doi.org/10.1016/j.msea.2007.07.002.

[29] Song KH, Fujii H, Nakata K. Effect of welding speed on microstructural and mechanical properties of friction stir welded Inconel 600. Materials \& Design. 2009;30(10):3972 3978. http://dx.doi.org/10.1016/j.matdes.2009.05.033.

[30] Lemos GVB, Hirsch TK, Rocha A, Nunes RM. Residual stress analysis of drive shafts after induction hardening. Materials Research. 2014;17(Supl 1):70-74. http://dx.doi.org/10.1590/ S1516-14392014005000077.

[31] Lemos GVB. Análise de tensões residuais em eixos automotivos do aço DIN 38B3 temperados por indução [dissertação de mestrado]. Porto Alegre: Universidade Federal do Rio Grande do Sul; 2012

[32] Lienert T, Stellwag W Jr, Lehman L, editors. Comparison of heat inputs: friction stir welding vs. arc welding. In: American Welding Society; 2002; Columbus, $\mathrm{OH}$. Columbus: Edison Welding Institute; 2002. p. 1-3.

[33] Rao D, Huber K, Heerens J, Santos JF, Huber N. Asymmetric mechanical properties and tensile behaviour prediction of aluminium alloy 5083 friction stir welding joints. Materials Science and Engineering A. 2013;565:44-50. http://dx.doi. org/10.1016/j.msea.2012.12.014.

[34] Lemos GVB, HankeS, Santos JF, Bergmann L, Reguly A, Strohaecker TR. Progress in friction stir welding of Ni alloys. Science and Technology of Welding and Joining. 2017;1-5. http://dx.doi. org/10.1080/13621718.2017.1288953.

[35] Meyers MA, Chawla KK. Mechanical behavior of materials. Cambridge: Cambridge University Press; 2009. p. 420-425. (vol. 2)

[36] Schwitzke M. Aerodynamische Anregung resonanter Schaufelschwingungen in Radialturbinen. Karlsruhe: Forschungsberichte aus dem Institut für Thermische Strömungsmaschinen; 2015. 